Modeling, Identification and Control, Vol. 29, No. 2, 2008, pp. 59-66

\title{
Operational space synchronization of two robot manipulators through a virtual velocity estimate
}

\author{
Erik Kyrkjebø ${ }^{1}$ Kristin Y. Pettersen ${ }^{2}$
}

\author{
${ }^{1}$ Department of Applied Cybernetics, SINTEF Information and Communication Technology, N-7465 Trondheim, \\ Norway. E-mail: Erik.Kyrkjebo@sintef.no \\ ${ }^{2}$ Department of Engineering Cybernetics, Norwegian University of Science and Technology, N-7491 Trondheim, \\ Norway. E-mail: Kristin.Y.Pettersen@itk.ntnu.no
}

\begin{abstract}
Two robot manipulators are synchronized in a leader-follower scheme where only joint position measurements of the leader are available. A virtual manipulator is designed to provide a velocity estimate of the unknown leader velocity to the control law of the follower. The closed-loop errors are shown to be uniformly globally practically asymptotically stable.*
\end{abstract}

Keywords: Robot manipulator, synchronization, observer, nonlinear, virtual

\section{Introduction}

Leader-follower synchronization control can be considered as a tracking control problem where the reference is a physical object with dynamics that is subject to disturbances and actuator limitations. For the particular case of a robot manipulator arm, the actual states of the leader manipulator may diverge from its ideal path due to external disturbances, unmodeled dynamics or friction phenomenas, or actuator saturations. Under these constraints we cannot guarantee that the leader manipulator tracks its desired path perfectly, and thus knowledge of the desired path of the reference may not be enough to assure synchronization in the leader-follower system. The synchronization control problem is often complicated by the fact that frequently only position measurements are available or reliable. In practice, robot manipulators are often equipped with high-precision position sensors

\footnotetext{
* (C) [2007] IEEE. Reprinted, with permission, from Erik Kyrkjebø and Kristin Y. Pettersen, "Operational space synchronization of two robot manipulators through a virtual velocity estimate", Proceedings of 46th IEEE Conference on Decision and Control, New Orleans, USA, December 2007.
}

such as encoders, but velocity or acceleration measurements are not so readily available, and are often contaminated with noise when obtained from low-quality tachometers or through numerical differentiation techniques. Model based observers utilize the nonlinear dynamic model of the manipulator to reconstruct velocity and acceleration information, and may produce estimates less contaminated by noise than simple differentiation techniques. Differentiation and model based estimation approaches to the problem of output trajectory control of robot manipulators can be found extensively in literature (Kelly, 1993; Loría and Ortega, 1995; De Queiroz et al., 1997; Loría and Melhem, 2002). However, the dynamic model of a robot manipulator is not always known, and thus alternative approaches to model based observers must be employed to estimate the velocity and acceleration of the manipulator. In the following, we propose to utilize the known kinematics of a manipulator with unknown dynamics to provide a velocity estimate available to the synchronization control law. The velocity estimate is constructed using a virtual manipulator that is stabilized to the leader manipulator through a kinematic control procedure. Hence, the available information (kinematics) is uti- 
lized to estimate the states of the leader manipulator, but the requirement of knowing the parameters of the more complex dynamic model is lifted.

Synchronization is the theory of time conformity between systems, and can be found both as a natural phenomenon in nature (Camazine et al., 2001) as well as the controlled synchronization of artificial systems as reported in Huygens (1673) for a pair of pendulum clocks, and later revisited by Blekhman (1971) in works on vibromechanics. It can be seen as a type of state cooperation among two or more (sub)systems, and has received increasing attention in the control community (Fradkov et al., 2000; Nijmeijer and Rodriguez-Angeles, 2003). The synchronization scheme can be divided into the coordinated synchronization scheme where an external reference is the synchronization goal, and an internal cooperative scheme where all participants are responsible for synchronization. Both a coordinated and a cooperative synchronization control scheme for robot manipulators were presented in Nijmeijer and Rodriguez-Angeles (2003) using a controller-observer scheme with only position measurements to estimate unmeasured state information. The coordinated leader-follower scheme (Nijmeijer and Rodriguez-Angeles, 2003; Rodriguez-Angeles and Nijmeijer, 2001) depends on a leader manipulator to govern the motion of the follower manipulator, and thus only the follower is responsible for applying the synchronization control action necessary to coordinate the two manipulators. This relieves the information requirements on the leader manipulator, and suggests that synchronization can be achieved between manipulators even though the parameters of the dynamical model of one of the manipulators are unknown. The coordinated synchronization approach of Rodriguez-Angeles and Nijmeijer (2001) was later used in Kyrkjebø and Pettersen (2003) to synchronize two ships in a leader-follower underway replenishment operation. The controller-observer approach closely interlinks the stability of both the controller and the observers, and it can thus be difficult and tedious to tune the control gains satisfactorily.

The virtual system design has been utilized both as an abstraction vehicle (Crowley, 1989; Salichs et al., 1991) and as an intermediate level between the desired trajectories of a system and the controller. The virtual system can be considered as a low-level controller in a two-level control structure (Fradkov et al., 1991; Gusev et al., 1998), and has been used in Sakaguchi et al. (1999) as the mapping of a physical vehicle on an entry-ramp on a main lane in order to do merging control of autonomous mobile robots, and in Egerstedt et al. (2001) to control a reference point on a planned path. The latter approach has been utilized in $\mathrm{Hu}$ et al.
(2003) to combine the task of path following and obstacle avoidance, and in Cheng et al. (2004) with a modified goal point to improve practical robustness to path diversity.

In the proposed observer-controller design of Nijmeijer and Rodriguez-Angeles (2003) and Kyrkjebø and Pettersen (2003), the stability analysis and practical tuning of the scheme is involved due to the inherent coupling in between the observers and controller. In order to facilitate both the tuning of the controller and the stability analysis in a leader-follower synchronization scheme, Kyrkjebø et al. (2006) proposed a virtual vehicle approach to the underway replenishment operation, which required no additional information on leader states or model parameters. In the following, we adopt the results of Kyrkjebø et al. (2006) to the $n$-degree-of-freedom control of robot manipulators in the operational (cartesian) space. The control scheme utilizes a virtual manipulator as a state estimator of the leader manipulator velocity and acceleration, and yields uniform global practical asymptotic stability (Chaillet, 2006) of the closed-loop errors, meaning asymptotic stability to a ball about the origin that can be made arbitrarily small by a suitable choice of gains.

We will first present the general system model and the problem statement and approach in Sect. 2, and then the virtual manipulator design in Sect. 3. The synchronization controller is presented in Sect. 4, stability is addressed in Sect. 5 while simulations are presented in Sect. 6. Final remarks and conclusions are reported in Sect. 7.

\section{Preliminaries}

We consider leader-follower synchronization control for fully actuated robot manipulators with $n \leq 6$ joints, where the only available measurement from the leader is the position vector. The leader robot is driven by an input torque $\tau_{m}$ that is designed to drive the operational space coordinates $\mathbf{x}_{m}, \dot{\mathbf{x}}_{m} \in \mathbb{R}^{m}$ to a desired trajectory $\mathbf{x}_{d}, \dot{\mathbf{x}}_{d} \in \mathbb{R}^{m}$. The input torque of the leader, as well as the dynamical model and its parameters, is considered unknown and thus prevents us from designing a model based observer for the leader. There is no guarantee that the leader follows the desired trajectory perfectly, and thus the follower cannot simply track the desired trajectory $\mathbf{x}_{d}, \dot{\mathbf{x}}_{d}$, but must synchronize its states $\mathbf{x}, \dot{\mathbf{x}}$ to the leader states $\mathbf{x}_{m}, \dot{\mathbf{x}}_{m}$ to achieve coordination.

In the following, the minimum and maximum eigenvalue of a positive definite matrix $\mathbf{M}$ will be denoted as $\mathbf{M}_{m}$ and $\mathbf{M}_{M}$, respectively. The norm of a vector $\mathbf{x}$ is defined as $\|\mathbf{x}\|=\sqrt{\mathbf{x}^{T} \mathbf{x}}$ and the induced norm of a matrix $\mathbf{M}$ is $\|\mathbf{M}\|=\max _{\|\mathbf{x}\|=1}\|\mathbf{M x}\|$. 


\subsection{System model and properties}

The dynamic model of a robot manipulator in the joint space $\mathbf{q} \in \mathbb{R}^{n}$ can be written as (Sciavicco and Siciliano, 1996)

$$
\mathbf{M}_{\mathbf{q}}(\mathbf{q}) \ddot{\mathbf{q}}+\mathbf{C}_{\mathbf{q}}(\mathbf{q}, \dot{\mathbf{q}}) \dot{\mathbf{q}}+\mathbf{f}_{\mathbf{q}}(\dot{\mathbf{q}})+\mathbf{g}_{\mathbf{q}}(\mathbf{q})=\tau_{\mathbf{q}}
$$

where $\mathbf{q}$ are generalized coordinates, $\mathbf{M}_{\mathbf{q}}(\mathbf{q})$ is the positive definite inertia matrix, $\mathbf{C}_{\mathbf{q}}(\mathbf{q}, \dot{\mathbf{q}})$ is the matrix of Coriolis and centripetal forces, $\mathbf{f}_{\mathbf{q}}(\dot{\mathbf{q}})$ is a general function of friction forces, and gravitational forces are given in $\mathbf{g}_{\mathbf{q}}(\mathbf{q})$. The differential kinematic relation

$$
\dot{\mathbf{x}}=\mathbf{J}(\mathbf{q}) \dot{\mathbf{q}}
$$

relates the joint space from the operational space $\mathbf{x} \in$ $\mathbb{R}^{m}$ of Khatib (1987). We will assume that $m$ is equal to the number of joints $n$, and thus the manipulator acts in nonsingular configurations and $\mathbf{x}$ constitutes a set of generalized coordinates. The dynamic model of the manipulator in the operational space can thus be written as Khatib (1987)

$$
\mathbf{M}(\mathbf{x}) \ddot{\mathbf{x}}+\mathbf{C}(\mathbf{x}, \dot{\mathbf{x}}) \dot{\mathbf{x}}+\mathbf{f}(\mathbf{x}, \dot{\mathbf{x}})+\mathbf{g}(\mathbf{x})=\tau
$$

where the inertia matrix $\mathbf{M}(\mathbf{x})$ is positive definite and the Coriolis and centripetal matrix $\mathbf{C}(\mathbf{x}, \dot{\mathbf{x}})$ is defined in terms of Christoffel symbols. The dissipative vector $\mathbf{f}(\mathbf{x}, \dot{\mathbf{x}})$ collects friction forces that are nonlinear in velocity and that may also depend on joint angles, while $\mathbf{g}(\mathbf{x})$ is the vector of gravitational forces. The control input vector $\tau$ is generalized forces and moments acting on the system. The model (3) is an Euler-Lagrange system (Kyrkjebø and Pettersen, 2005), and has a number of properties (Ortega and Spong, 1989)

$\mathbf{P 1}$ The positive definite true inertia matrix $\mathbf{M}(\mathbf{x})$ satisfy $0<\mathbf{M}_{m} \leq\|\mathbf{M}(\mathbf{x})\| \leq \mathbf{M}_{M}<\infty$, where $\mathbf{M}_{m}$ and $\mathbf{M}_{M}$ are positive constants.

P2 The inertia matrix $\mathbf{M}(\mathbf{x})$ is differentiable in $\mathbf{x}$ and $\mathbf{y}^{T}(\dot{\mathbf{M}}(\mathbf{x})-2 \mathbf{C}(\mathbf{x}, \dot{\mathbf{x}})) \mathbf{y}=0, \forall \mathbf{x}, \mathbf{y} \in \mathbb{R}^{n}$.

P3 The Coriolis term in Christoffel symbols satisfies $\mathbf{C}(\mathbf{x}, \mathbf{y}) \mathbf{z}=\mathbf{C}(\mathbf{x}, \mathbf{z}) \mathbf{y}$, and also $\|\mathbf{C}(\mathbf{x}, \dot{\mathbf{x}})\| \leq$ $\mathbf{C}_{M}\|\dot{\mathbf{x}}\|$.

We will also make the following assumption on the dissipation vector $\mathbf{f}(\mathbf{x}, \dot{\mathbf{x}}) \dot{\mathbf{x}}$

Assumption 1 The dissipation vector $\mathbf{f}(\mathbf{x}, \dot{\mathbf{x}})$ is linear in velocity and lower bounded by a non-negative constant $\mathbf{F}_{m}$;

$$
\mathbf{f}(\mathbf{x}, \dot{\mathbf{x}})=\mathbf{F}(\mathbf{x}), \quad\|\mathbf{F}(\mathbf{x})\| \geq \mathbf{F}_{m} \geq 0 .
$$

Thus, we restrict the friction in the system to linear (viscous) friction.
The relationship between joint angles $\mathbf{q}$ and operational space coordinates $\mathbf{x}$ is known through the direct kinematics equation. In this paper, we will assume that the kinematic relationships for both manipulators are known.

\section{Assumption 2 The direct kinematic equation}

$$
\mathbf{x}=\mathbf{d}(\mathbf{q})
$$

is known for both manipulators. The nonlinear function $\mathbf{d}(\cdot)$ allows computation of the operational space variables from knowledge of the joint space variables.

Note that the proposed virtual manipulator design of this section coordinates two robot manipulators in the operational space, and not in the joint space as in Rodriguez-Angeles and Nijmeijer (2001) and Bondhus et al. (2004). Hence, we formulate the coordination scheme in the task space of the robot manipulator, which is more suitable to applications where the robot manipulators should follow a geometrically specified motion, or in applications where two robots should manipulate large rigid structures. Thus, we inherently assume that the image of the forward kinematics of the leader must be contained within the image of the forward kinematics of the follower. Note also that if measurements of the operational space position variables $\mathbf{x}$ and $\mathbf{x}_{m}$ are available, the virtual manipulator design does not require that the direct kinematic function $\mathbf{f}(\cdot)$ is known explicitly.

\subsection{Problem statement}

The problem of synchronizing two robot manipulators are considered in the operational (cartesian) space coordinates. The manipulators are fully actuated, and the number of joints (all revolute) $n$ are equal to the number of degrees of freedom $m$ in the operational space.

The dynamic model of the leader manipulator with mass and inertia parameters is considered unknown, as well as the velocity and acceleration of the leader manipulator. The position and orientation of the leader manipulator $\mathbf{q}_{m}$ is known and measured, and the kinematic equation $\mathbf{x}_{m}=\mathbf{f}_{m}\left(\mathbf{q}_{m}\right)$ and the differential kinematics relationship through the Jacobian matrix $\mathbf{J}_{m}\left(\mathbf{q}_{m}\right)$ is considered known. No knowledge of the desired trajectory of the leader is assumed.

The parameters of the dynamic model of the follower manipulator are considered known, as well as its position and orientation vector $\mathbf{q}$. We assume that the velocity vector $\dot{\mathbf{q}}$, the kinematic relationship $\mathbf{f}(\mathbf{q})$ and differential kinematic relationship through the Jacobian matrix $\mathbf{J}(\mathbf{q})$ in (2) are known for the follower manipulator. In practice, this relates to the problem of synchronizing a known robot manipulator to an unknown 


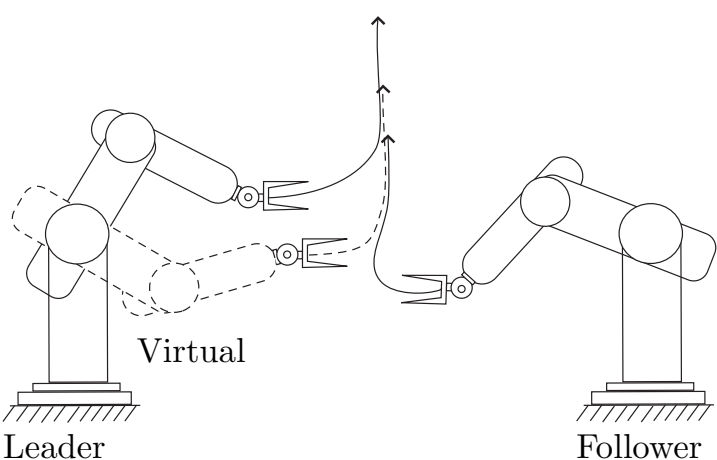

Figure 1: Leader, follower and virtual manipulator

robot manipulator where only the length and type of the joints are known, and where only the joint position and orientation vector $\mathbf{q}_{m}$ is measured. However, the virtual manipulator approach is easily adopted to the design in Kyrkjebø and Pettersen (2006) where the restriction of knowing the velocity of the follower manipulator is lifted.

\subsection{Problem approach}

To solve the synchronization problem without velocity measurements of the leader, we will utilize the concept of a virtual manipulator that stabilizes itself to the leader manipulator, see Figure 1. This virtual manipulator is based on the differential kinematic relationship of the leader through the Jacobian relationship

$$
\mathbf{x}_{v}=\mathbf{J}_{m}\left(\mathbf{q}_{v}\right) \dot{\mathbf{q}}_{v}
$$

and uses the virtual joint velocity $\dot{\mathbf{q}}_{v}$ as a control input. In this paper, we adopt the leader manipulator as a motion reference for the follower manipulator to achieve $(\mathbf{x}, \dot{\mathbf{x}}) \rightarrow\left(\mathbf{x}_{m}, \dot{\mathbf{x}}_{m}\right)$, and thus the two manipulators will perform the same movement in the operational space.

\section{Virtual manipulator design}

The only measurement available from the leader is the joint position vector $\mathbf{q}_{m}$ which translates directly into the position and orientation vector $\mathbf{x}_{m}$ through the relationship in (5). Since we have no information about the parameters of the dynamic model or of the control input to the leader, we are precluded from designing a model-based observer for the leader states. We propose to design a virtual manipulator as an intermediate controlled manipulator that is stabilized to the leader manipulator based only on position measurement feedback.
As in Gusev et al. (1998), in the first step (kinematic level) we consider the velocities $\dot{\mathbf{q}}_{v}$ of the virtual manipulator as the control inputs, and design them in such a way that we ensure convergence of the virtual trajectories to the leader trajectories. In a way, we can consider the trajectories $\mathbf{x}_{v}$ and velocities $\dot{\mathbf{q}}_{v}$ as estimates of $\mathbf{x}_{m}$ and $\dot{\mathbf{q}}_{m}$, that is, the virtual manipulator is a form of kinematic estimator of the leader states through the position feedback loop.

The virtual manipulator is defined by its differential kinematic model in (6), and based on practical considerations we will assume that the leader velocity is bounded.

Assumption 3 The velocity of the leader manipulator is bounded as

$$
\sup _{t}\left\|\dot{\mathbf{q}}_{m}\right\|=V_{M}<\infty
$$

with a known upper bound $V_{M}$.

We can define the virtual manipulator tracking errors in the operational space as

$$
\mathbf{e}_{v}=\mathbf{x}_{v}-\mathbf{x}_{m}=\mathbf{d}_{m}\left(\mathbf{q}_{v}\right)-\mathbf{d}_{m}\left(\mathbf{q}_{m}\right)
$$

and

$$
\dot{\mathbf{e}}_{v}=\dot{\mathbf{x}}_{v}-\dot{\mathbf{x}}_{m}=\mathbf{J}_{m}\left(\mathbf{q}_{v}\right) \dot{\mathbf{q}}_{v}-\mathbf{J}_{m}\left(\mathbf{q}_{m}\right) \dot{\mathbf{q}}_{m} .
$$

We propose the following virtual control law

$$
\dot{\mathbf{q}}_{v}=-\mathbf{J}_{m}^{-1}\left(\mathbf{q}_{v}\right) \mathbf{L}_{1} \mathbf{e}_{v}-\mathbf{J}_{m}^{-1}\left(\mathbf{q}_{v}\right) \mathbf{L}_{2} \mathbf{z}
$$

where $\mathbf{L}_{1}$ and $\mathbf{L}_{2}$ are symmetric positive gain matrices, and

$$
\dot{\mathbf{z}}=\mathbf{e}_{v}
$$

to add an integral term. The closed-loop equations can be written in the following form

$$
\dot{\mathbf{e}}_{v}=-\mathbf{L}_{1} \mathbf{e}_{v}-\mathbf{L}_{2} \mathbf{z}-\mathbf{J}_{m}\left(\mathbf{q}_{m}\right) \dot{\mathbf{q}}_{m} .
$$

Theorem 1 The closed-loop error dynamics of system (6) satisfying Assumption 3, with the control law (10), are uniformly globally practically asymptotically stable.

Proof 1 Consider the following Lyapunov function candidate

$$
V_{v}\left(\mathbf{z}, \mathbf{e}_{v}\right)=\frac{1}{2} \mathbf{e}_{v}^{T} \mathbf{e}_{v}+\frac{1}{2} \mathbf{z}^{T} \mathbf{L}_{2} \mathbf{z}+\frac{1}{2} \mathbf{z}^{T} \mathbf{e}_{v}
$$

which is positive definite for $\mathbf{L}_{2, m}>1 / 4$. Differentiating along the closed-loop trajectories we get

$$
\begin{aligned}
\dot{V}_{v}\left(\mathbf{z}, \mathbf{e}_{v}\right)= & -\mathbf{e}_{v}^{T}\left(\mathbf{L}_{1}-\frac{1}{2} \mathbf{I}\right) \mathbf{e}_{v}-\frac{1}{2} \mathbf{z}^{T} \mathbf{L}_{2} \mathbf{z}-\frac{1}{2} \mathbf{z}^{T} \mathbf{L}_{1} \mathbf{e}_{v} \\
& -\left(\mathbf{e}_{v}^{T}+\frac{1}{2} \mathbf{z}^{T}\right) \mathbf{J}_{m}\left(\mathbf{q}_{m}\right) \dot{\mathbf{q}}_{m}
\end{aligned}
$$


Using (7), and the relation $2|a b| \leq\left(\lambda a^{2}+b^{2} / \lambda\right)$ for any real $a, b$ and any positive $\lambda$, it follows that

$$
\begin{aligned}
\dot{V}_{v}\left(\mathbf{z}, \mathbf{e}_{v}\right) \leq & -\left(\mathbf{L}_{1, m}-\frac{1}{2}-\frac{\lambda}{4} \mathbf{L}_{1, M}-\frac{3 V_{M}}{2\left\|\left(\mathbf{e}_{v}, \mathbf{z}\right)\right\|}\right)\left\|\mathbf{e}_{v}\right\|^{2} \\
& -\frac{1}{2}\left(\mathbf{L}_{2, m}-\frac{1}{2 \lambda} \mathbf{L}_{1, M}-\frac{3 V_{M}}{\left\|\left(\mathbf{e}_{v}, \mathbf{z}\right)\right\|}\right)\|\mathbf{z}\|^{2}
\end{aligned}
$$

since $\left\|\mathbf{J}\left({ }_{m} \cdot\right)\right\| \leq 1$, and where $\lambda$ designates any positive constant. We design the gain matrices $\mathbf{L}_{1}$ and $\mathbf{L}_{2}$ in such a way that $\mathbf{L}_{i, M} \leq \ell \mathbf{L}_{i, m}, i \in\{1,2\}$, for some $\ell>0$. Then, letting $\lambda=2 / \ell$ and $\delta_{v}$ be any given positive constant, we can see that any gain matrices satisfying

$$
\begin{aligned}
& \mathbf{L}_{1, m}=3+\frac{3 V_{M}}{\delta_{v}} \\
& \mathbf{L}_{2, m}=2+\frac{3 \ell^{2}}{4}+\left(1+\frac{\ell^{2}}{4}\right) \frac{3 V_{M}}{2 \delta_{v}}
\end{aligned}
$$

generate the following bound of the derivative of $V_{v}$ :

$$
\left\|\mathbf{e}_{v}\right\|^{2}+\|\mathbf{z}\|^{2} \geq \delta_{v}^{2} \Rightarrow \dot{V}_{v}\left(\mathbf{z}, \mathbf{e}_{v}\right) \leq-\left\|\mathbf{e}_{v}\right\|^{2}-\|\mathbf{z}\|^{2} .
$$

Note that $V_{v}$ is positive definite and radially unbounded for this choice of gains. Due to the linear dependency of $\mathbf{L}_{1, m}$ and $\mathbf{L}_{2, m}$ in $1 / \delta_{v}$, we conclude using the results in Chaillet and Loría (2005) that (11-12) is uniformly practically asymptotically stable, which implies that the region to which solutions converge - from any initial condition - can be reduced as much as desired by enlarging $\mathbf{L}_{1, m}$ and $\mathbf{L}_{2, m}$.

\section{Follower vehicle design}

Using the velocity information from the virtual manipulator design of Sect. 3, we can design a synchronization controller for the follower manipulator to follow the virtual manipulator of (6). Note that the joint velocity $\dot{\mathbf{q}}_{v}$ is now known through the definition of the control law of (10), and through the differential kinematic relationship of (6) we can obtain the velocity $\dot{\mathbf{x}}_{v}$ of the virtual manipulator. Furthermore, due to our design of the virtual controller (10), we can also obtain an expression for the acceleration of the virtual manipulator which will be partially available for control purposes. In our synchronization approach, we will assume that the velocity of the follower manipulator is measured and known.

The variables available from the virtual manipulator design to the synchronization controller are

$$
\begin{aligned}
\dot{\mathbf{x}}_{v} & =\mathbf{J}_{m}\left(\mathbf{q}_{v}\right) \dot{\mathbf{q}}_{v}=-\mathbf{L}_{1} \mathbf{e}_{v}-\mathbf{L}_{2} \mathbf{z} \\
\ddot{\mathbf{x}}_{v} & =-\mathbf{L}_{1} \dot{\mathbf{e}}_{v}-\mathbf{L}_{2} \mathbf{e}_{v} \\
& =\left(\mathbf{L}_{1}^{2}-\mathbf{L}_{2}\right) \mathbf{e}_{v}+\mathbf{L}_{1} \mathbf{L}_{2} \mathbf{z}+\mathbf{L}_{1} \mathbf{J}_{m}\left(\mathbf{q}_{m}\right) \dot{\mathbf{q}}_{m} .
\end{aligned}
$$

Define the synchronization errors as

$$
\mathbf{e}=\mathbf{x}-\mathbf{x}_{v}, \quad \dot{\mathbf{e}}=\dot{\mathbf{x}}-\dot{\mathbf{x}}_{v}, \quad \ddot{\mathbf{e}}=\ddot{\mathbf{x}}-\ddot{\mathbf{x}}_{v} .
$$

Using the sliding surface from Slotine and Li (1987) as a passive filtering of the virtual vehicle states, we can design a virtual reference trajectory as

$$
\begin{array}{ll}
\dot{\mathbf{y}}_{v}= & \dot{\mathbf{x}}_{v}-\mathbf{\Lambda} \mathbf{e} \\
\ddot{\mathbf{y}}_{v}= & \ddot{\mathbf{x}}_{v}-\Lambda \dot{\mathbf{e}}
\end{array}
$$

where $\boldsymbol{\Lambda}>0$ is a design parameter. Let us denote

$$
\ddot{\mathbf{y}}_{v}^{\prime}=\left(\mathbf{L}_{1}^{2}-\mathbf{L}_{2}\right) \mathbf{e}_{v}+\mathbf{L}_{1} \mathbf{L}_{2} \mathbf{z}-\mathbf{\Lambda} \dot{\mathbf{e}}
$$

and thus

$$
\ddot{\mathbf{y}}_{v}=\ddot{\mathbf{y}}_{v}^{\prime}+\mathbf{L}_{1} \mathbf{J}_{m}\left(\mathbf{q}_{m}\right) \dot{\mathbf{q}}_{m}
$$

where $\ddot{\mathbf{y}}_{v}^{\prime}$ is available for control design. Defining

$$
\mathbf{s}=\dot{\mathbf{x}}-\dot{\mathbf{y}}_{v}=\dot{\mathbf{e}}+\Lambda \mathbf{e}
$$

as a measure of tracking, and using the relationship $\dot{\mathbf{x}}=\mathbf{s}-\dot{\mathbf{y}}_{v}$ we can rewrite (3) as

$$
\begin{aligned}
\mathbf{M}(\mathbf{x}) \dot{\mathbf{s}}= & -\mathbf{C}(\mathbf{x}, \dot{\mathbf{x}}) \mathbf{s}-\mathbf{F}(\mathbf{x}) \mathbf{s}+\tau \\
& -\mathbf{M}(\mathbf{x}) \ddot{\mathbf{y}}_{v}-\mathbf{C}(\mathbf{x}, \dot{\mathbf{x}}) \dot{\mathbf{y}}_{v}-\mathbf{F}(\mathbf{x}) \dot{\mathbf{y}}_{v}-\mathbf{g}(\mathbf{x})
\end{aligned}
$$

We propose the following control law

$\tau=\mathbf{M}(\mathbf{x}) \ddot{\mathbf{y}}_{v}^{\prime}+\mathbf{C}(\mathbf{x}, \dot{\mathbf{x}}) \dot{\mathbf{y}}_{v}+\mathbf{F}(\mathbf{x}) \dot{\mathbf{y}}_{v}+\mathbf{g}(\mathbf{x})-\mathbf{K}_{d} \mathbf{s}-\mathbf{K}_{p} \mathbf{e}$

where $\mathbf{K}_{p}$ and $\mathbf{K}_{d}$ are symmetric positive gain matrices. Consider the following Lyapunov function candidate

$$
V_{e}(\mathbf{e}, \mathbf{s})=\frac{1}{2} \mathbf{s}^{T} \mathbf{M}(\mathbf{x}) \mathbf{s}+\frac{1}{2} \mathbf{e}^{T} \mathbf{K}_{p} \mathbf{e}
$$

Differentiating along the closed-loop trajectories we get

$$
\begin{aligned}
\dot{V}_{e}(\mathbf{e}, \mathbf{s})= & -\mathbf{s}^{T}\left[\mathbf{F}(\mathbf{x})+\mathbf{K}_{d}\right] \mathbf{s}-\mathbf{e}^{T} \boldsymbol{\Lambda}^{T} \mathbf{K}_{p} \mathbf{e} \\
& -\mathbf{s}^{T} \mathbf{M}(\mathbf{x}) \mathbf{L}_{1} \mathbf{J}_{m}\left(\mathbf{q}_{m}\right) \dot{\mathbf{q}}_{m} .
\end{aligned}
$$

Let $\delta_{e}$ be any given positive constant. Then, it holds that, for all $\|\mathbf{e}\|^{2}+\|\mathbf{s}\|^{2} \geq \delta_{e}^{2}$,

$$
\begin{aligned}
\dot{V}_{e}(\mathbf{e}, \mathbf{s}) & \leq\left[\mathbf{F}_{m}+\mathbf{K}_{d, m}-\frac{1}{2 \delta_{e}} \mathbf{M}_{M} \mathbf{L}_{1, M}\right]\|\mathbf{s}\|^{2} \\
& -\left[\boldsymbol{\Lambda}_{m} \mathbf{K}_{p, m}-\frac{1}{2 \delta_{e}} \mathbf{M}_{M} \mathbf{L}_{1, M}\right]\|\mathbf{e}\|^{2}
\end{aligned}
$$

Proceeding as in Section 3 and observing that the choice of $\mathbf{K}_{d, m}$ and $\mathbf{K}_{p, m}$ can be made as an affine function of $1 / \delta_{e}$, we can conclude uniform global practical asymptotic stability. 


\section{Stability analysis of the overall system}

The control law of the follower synchronizes the follower manipulator to the virtual manipulator based on a computed virtual reference velocity, and the virtual manipulator is in turn stabilized to the leader manipulator through the virtual control law.

Theorem 2 Consider the robot manipulator model (3) satisfying Properties 1-3, the virtual control law (10) and the synchronization controller (28). Under Assumptions 1-3, the overall closed-loop system is uniformly globally practically asymptotically stable.

Proof 2 Take as a positive definite Lyapunov function candidate

$$
V(\widetilde{\eta})=\frac{1}{2} \widetilde{\eta}^{T} \mathbf{P} \widetilde{\eta}
$$

where

$$
\widetilde{\eta}=\left[\mathbf{e}^{T}, \mathbf{s}^{T}, \mathbf{z}^{T}, \mathbf{e}_{v}^{T}\right]^{T}
$$

and

$$
\mathbf{P}=\left[\begin{array}{cccc}
\mathbf{K}_{p} & 0 & 0 & 0 \\
0 & \mathbf{M}(\mathbf{x}) & 0 & 0 \\
0 & 0 & \mathbf{L}_{2} & \frac{1}{2} \mathbf{I} \\
0 & 0 & \frac{1}{2} \mathbf{I} & \mathbf{I}
\end{array}\right]
$$

is a composition of the Lyapunov functions (13) and (29) of Sect. 3 and 4. Differentiating along trajectories yields

$$
\dot{V}(\widetilde{\eta})=\quad-\widetilde{\eta}^{T} \mathbf{Q} \widetilde{\eta}+\beta\left(\mathbf{s}, \mathbf{z}, \mathbf{e}_{v}, \dot{\mathbf{q}}_{m}\right)
$$

where

$$
\mathbf{Q}=\left[\begin{array}{cccc}
\boldsymbol{\Lambda}^{T} \mathbf{K}_{p} & 0 & 0 & 0 \\
0 & \mathbf{F}(\mathbf{x})+\mathbf{K}_{d} & 0 & 0 \\
0 & 0 & \frac{1}{2} \mathbf{L}_{2} & \frac{1}{4} \mathbf{L}_{1} \\
0 & 0 & \frac{1}{4} \mathbf{L}_{1} & \mathbf{L}_{1}-\frac{1}{2} \mathbf{I}
\end{array}\right]
$$

and

$$
\begin{aligned}
\beta\left(\mathbf{s}, \mathbf{z}, \mathbf{e}_{v}, \dot{\mathbf{q}}_{m}\right)= & -\mathbf{s}^{T} \mathbf{M}(\mathbf{x}) \mathbf{L}_{1} \mathbf{J}_{m}\left(\mathbf{q}_{m}\right) \dot{\mathbf{q}}_{m} \\
& -\frac{1}{2} \mathbf{z}^{T} \mathbf{J}_{m}\left(\mathbf{q}_{m}\right) \dot{\mathbf{q}}_{m}-\mathbf{e}_{v}^{T} \mathbf{J}_{m}\left(\mathbf{q}_{m}\right) \dot{\mathbf{q}}_{m}
\end{aligned}
$$

Let $\delta$ be any given positive constant, and we have the following property

$\|\widetilde{\eta}\| \geq \delta \Rightarrow$

$\left\|\beta\left(\mathbf{s}, \mathbf{z}, \mathbf{e}_{v}, \dot{\mathbf{q}}_{m}\right)\right\| \leq \frac{V_{M}}{\delta}\left(\mathbf{M}_{M} \mathbf{L}_{1, M}\|\mathbf{s}\|^{2}+\frac{\|\mathbf{z}\|^{2}}{2}+\frac{\left\|\mathbf{e}_{v}\right\|^{2}}{2}\right)$
Consequently, in view of (15) and (31), and repeating a similar reasoning while choosing the minimum eigenvalue of the gain matrices $\mathbf{K}_{p}, \mathbf{K}_{d}, \mathbf{L}_{1}$ and $\mathbf{L}_{2}$ large enough, it holds that

$$
\dot{V}(\widetilde{\eta}) \leq-\|\widetilde{\eta}\|^{2}, \quad \forall\|\widetilde{\eta}\| \geq \delta .
$$

Since the dependency on the bound on $\beta$ (and on the gain matrices) in $1 / \delta$ is again affine, uniform global practical asymptotic stability of $\widetilde{\eta}$ follows.

\section{Simulation study}

The operational space synchronization scheme with virtual velocity estimates was tested in a simulation environment in MATLAB using a two-link manipulator structure from Sciavicco and Siciliano (1996). The leader manipulator tracked an operational space rectilinear path from $\mathbf{x}_{d}(0)=[0.2,0.2]^{T}$ to $\mathbf{x}_{d}\left(t_{f}\right)=$ $[0.1,-0.6]^{T}$ with a trapezoidal velocity profile and a trajectory duration of $t_{f}=25 \mathrm{~s}$. The maximum velocity was restricted to $1 \mathrm{~m} / \mathrm{s}$, and an inverse dynamic trajectory tracking scheme in the operational space was employed for the leader manipulator.

The leader robot parameters were taken from Sciavicco and Siciliano (1996, Section 6.7) as $a_{1}=a_{2}=1$ $\mathrm{m}, l_{1}=l_{2}=0.5 \mathrm{~m}, m_{l_{1}}=m_{l_{2}}=50 \mathrm{~kg}, I_{l_{1}}=I_{l_{2}}=10$ $\mathrm{kg} \cdot \mathrm{m}^{2}, k_{r_{1}}=k_{r_{2}}=100, m_{m_{1}}=m_{m_{2}}=5 \mathrm{~kg}$, and $I_{m_{1}}=I_{m_{2}}=0.001 \mathrm{~kg} \cdot \mathrm{m}^{2}$. Data for the two equal joint actuators were chosen as $F_{m_{1}}=F_{m_{2}}=0.001$ $\mathrm{N} \cdot \mathrm{m} \cdot \mathrm{s} / \mathrm{rad}, R_{a_{1}}=R_{a_{2}}=10 \mathrm{ohm}, k_{t_{1}}=k_{t_{2}}=2$ $\mathrm{N} \cdot \mathrm{m} / \mathrm{A}$, and $k_{v_{1}}=k_{v_{2}}=2 \mathrm{~V} \cdot \mathrm{s} / \mathrm{rad}$. The control gains of the leader trajectory tracking controller were $\mathbf{K}_{p}=200 \mathbf{I}$ and $\mathbf{K}_{d}=150 \mathbf{I}$.

The follower parameters were chosen equal to the leader parameters, apart from $a_{1}=a_{2}=1.2 \mathrm{~m}$. The control gains were chosen as $\mathbf{L}_{1}=1 \mathbf{I}, \mathbf{L}_{2}=1 \mathbf{I}$ for the virtual manipulator, and $\mathbf{K}_{p}=700 \mathbf{I}, \mathbf{K}_{d}=450 \mathbf{I}$ for the follower manipulator using a sliding surface gain of $\boldsymbol{\Lambda}=0.1 \mathbf{I}$. Plots of the errors are shown in Figure 2, and the initial states were chosen as $\mathbf{x}(0)=[0.5,0.5]^{T}$ for the follower, $\mathbf{x}_{v}(0)=[0.9,0.0]^{T}$ for the virtual manipulator and as $\mathbf{x}_{m}(0)=[0.7,0.2]^{T}$ for the leader to illustrate convergence.

The virtual manipulator control errors $\mathbf{e}_{\nu}=\mathbf{x}_{v}-\mathbf{x}_{m}$, the coordination control errors $\mathbf{e}=\mathbf{x}-\mathbf{x}_{v}$ and the overall control errors $\mathbf{x}-\mathbf{x}_{r}$ are seen in Figure 2 to be practically asymptotically stable. Small oscillations in the virtual velocity errors are observed due to the unknown velocity of the leader manipulator, but due to the practical stability property of the closed-loop system the magnitude of these oscillations can be arbitrarily reduced within control saturation limits by enlarging the control gains. Simulations when only utilizing an approximately known model of the follower by assuming 

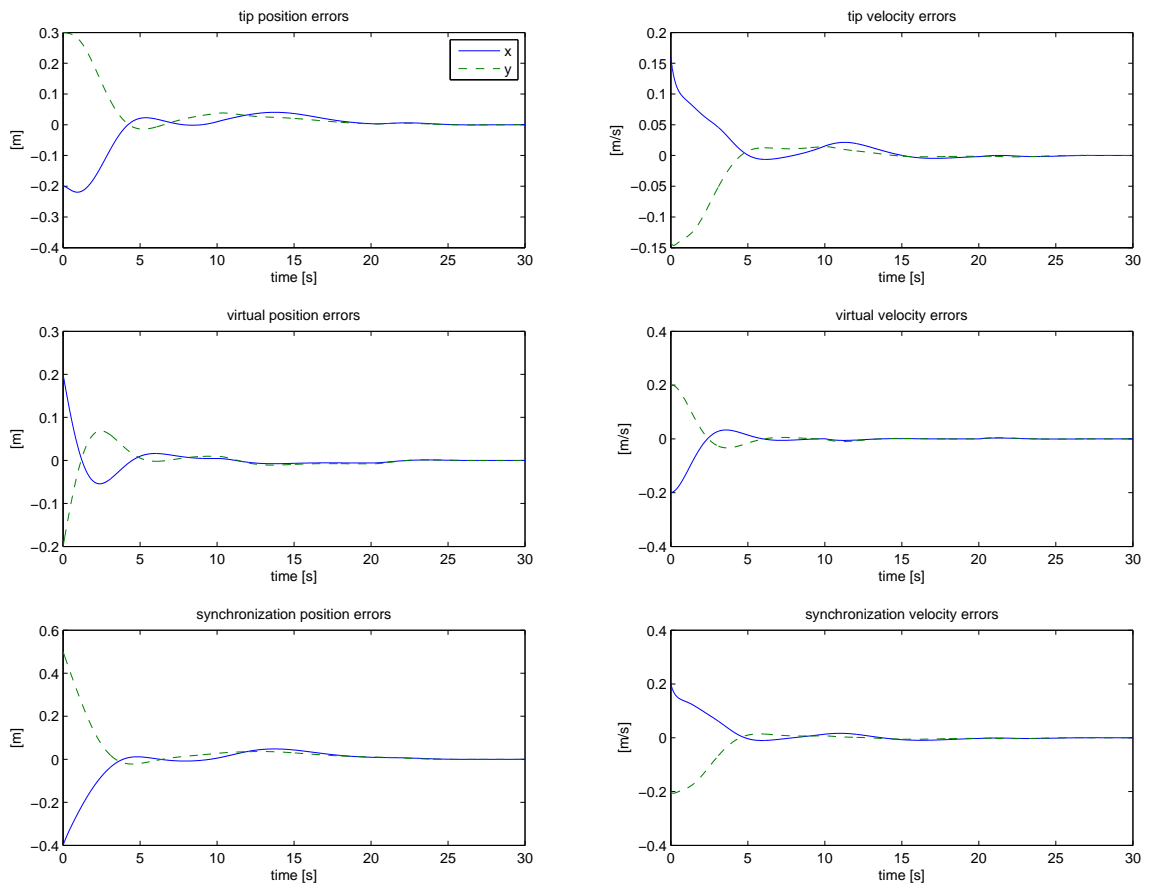

Figure 2: The total errors $\mathbf{x}-\mathbf{x}_{m}$ in the upper row, the virtual manipulator control errors $\mathbf{x}_{v}-\mathbf{x}_{m}$ in the middle row, and the coordination errors $\mathbf{x}-\mathbf{x}_{v}$ in the lower row, with positions $[\mathrm{m}]$ on the left and velocities $[\mathrm{m} / \mathrm{s}]$ on the right.

that the friction term $\mathbf{F}(\mathbf{x})$ is zero show similar results as illustrated in Figure 2, but are left out due to space constraints.

\section{Concluding remarks}

We have presented a control design approach to operational space synchronization of two robot manipulators were the velocity of the leader robot is unknown. The use of a virtual manipulator as a velocity estimator for the leader separates the state estimator from the synchronization controller design, thus allowing for a more intuitive gain tuning than for a closely linked observercontroller scheme. The overall control scheme is shown to be uniformly globally practically asymptotically stable.

Future work aims towards testing the scheme experimentally to investigate the behaviour and performance in a realistic environment.

\section{Acknowledgments}

This work was partially supported by the Norwegian Research Council under grant 159556/130.

\section{References}

Blekhman, I. Synchronization of Dynamical Systems. in Russian, English translation in ASME Press, New York: Synchronization in Science and Technology, Nauka, Moscow, 1971.

Bondhus, A. K., Pettersen, K. Y., and Nijmeijer, H. Master-slave synchronization of robot manipulators. In Proc. 6th IFAC Symp. on Nonlinear Control Systems. Stuttgart, Germany, 2004 pages 591 - 596 .

Camazine, S., Deneubourg, J.-L., Franks, N. R., Sneyd, J., Theraulaz, G., and Bonabeau, E. SelfOrganization in Biological Systems. Princeton studies in complexity. Princeton University Press, 2001.

Chaillet, A. Staiblité et Robustesse des Cascades Nonlinéaires et Application aux Systèmes Mécaniques. Ph.D. thesis, L'Université Paris XI Orsay, Supélec, France, 2006.

Chaillet, A. and Loría, A. Uniform semiglobal asymptotic stability for time-varying nonlinear cascaded systems. In Proc. IFAC World Congress. 2005.

Cheng, G., Gu, J., Bai, T., and Majdalawieh, O. A new efficient control algorithm using potential field: extension to robot path tracking. In Canadian Conf. 
on Electrical and Computer Engineering. 2004 pages $2035-2040$

Crowley, J. Asynchronous control of orientation and displacement in a robot vehicle. In Proc. 1989 IEEE Int. Conf. on Robotics and Automation. Scottsdale, AZ, USA, 1989 pages $1277-1282$.

De Queiroz, M., Hu, J., Dawson, D., and Burg, S., T.and Donepudi. Adaptive position/force control of robot manipulators without velocity measurements: theory and experimentation. IEEE Trans. on Systems, Man and Cybernetics, Part B, 1997. 27(5):796 -809 .

Egerstedt, M., Hu, X., and Stotsky, A. Control of mobile platforms using a virtual vehicle approach. IEEE Trans. on Automatic Control, 2001. 46(11):1777 1782 .

Fradkov, A., Gusev, S., and Makarov, I. Robust speedgradient adaptive control algorithms for manipulators and mobile robots. In Proc. 30th IEEE Conf. on Decision and Control. Brighton, England, 1991 pages $3095-3096$.

Fradkov, A. L., Nijmeijer, H., and Pogromsky, A. Y. Controlling Chaos and Bifurcations in Engineering Systems, chapter Adaptive observer-based synchronization, pages 417 - 438. CRC Press, 2000.

Gusev, S., Makarov, I., Paromtchik, I., Yakubovich, V., and Laugier, C. Adaptive motion control of a noholonomic vehicle. In Proc. 1998 IEEE Int. Conf. on Robotics and Automation. 1998 pages 3285 - 3290.

Hu, X., Alarcon, D., and Gustavi, T. Sensor-based navigation coordination for mobile robots. In Proc. 42nd IEEE Conf. on Decision and Control, 2003. 2003 pages $6375-6380$.

Huygens, C. Horoloquium Oscilatorium. Paris, France, 1673.

Kelly, R. A simple set-point robot controller by using only position measurements. In Proc. IFAC World Congress, volume 6. Sydney, Australia, 1993 pages $173-176$.

Khatib, O. A unified approach for motion and force control of robot manipulators: The operational space formulation. IEEE Journal of Robotics and Automation, 1987. RA-3(1):43 - 53 .

Kyrkjebø, E., Panteley, E., Chaillet, A., and Pettersen, K. Y. Group Coordination and Cooperative Control, volume 336 of Lecture Notes in Control and Information Systems, chapter A Virtual Vehicle Approach to
Underway Replenishment, pages 171 - 189. Springer Verlag, Troms $\varnothing$, Norway, 2006.

Kyrkjebø, E. and Pettersen, K. Y. Ship replenishment using synchronization control. In Proc. 6th IFAC Conf. on Manoeuvring and Control of Marine Craft. Girona, Spain, 2003 pages 286-291.

Kyrkjebø, E. and Pettersen, K. Y. Output synchronization control of Euler-Lagrange systems with nonlinear damping terms. In Proc. 44th IEEE Conf. on Decision and Control and European Control Conf. Sevilla, Spain, 2005 pages 4951 - 4957.

Kyrkjebø, E. and Pettersen, K. Y. A virtual vehicle approach to output synchronization control. In Proc. 45th Conf. on Decision and Control. San Diego, USA, 2006 .

Loría, A. and Melhem, K. Position feedback global tracking control of el systems: A state transformation approach. IEEE Trans. on Automatic Control, 2002. $47(5): 841-847$.

Loría, A. and Ortega, R. On tracking control of rigid and flexible joints robots. Appl Math Comp Sci, special issue on Mathematical Methods in Robotics, 1995. 5(2):101 - 113 .

Nijmeijer, H. and Rodriguez-Angeles, A. Synchronization of Mechanical Systems, volume 46. World Scientific Series on Nonlinear Science, Series A, 2003.

Ortega, R. and Spong, M. W. Adaptive motion control of rigid robots: A tutorial. Automatica, 1989. $25(6): 877-888$.

Rodriguez-Angeles, A. and Nijmeijer, H. Coordination of two robot manipulators based on position measurements only. Int. Journal of Control, 2001. $74: 1311-1323$.

Sakaguchi, T., Uno, A., and Tsugawa, S. Inter-vehicle communications for merging control. In Proc. IEEE Int. Vehicle Electronics Conf. 1999 pages $365-370$.

Salichs, M., Puente, E., Gachet, D., and Moreno, L. Trajectory tracking for a mobile robot - an application to contour following. In Proc. Int. Conf. on Industrial Electronics, Control and Instrumentation. Kobe, 1991 pages 1067 - 1070.

Sciavicco, L. and Siciliano, B. Modeling and Control of Robot Manipulators. McGraw-Hill Series in Electrical and Computer Engineering. McGraw-Hill, 1996.

Slotine, J.-J. E. and Li, W. Adaptive manipulator control a case study. In Proc. 1987 IEEE Int. Conf. on Robotics and Automation. 1987 pages $1392-1400$. 